\title{
Tratamiento de dolor lumbar crónico mediante radiofrecuencia pulsada y aplicación de esteroides em forma epidural. Estudio comparativo
}

\author{
Tratamento da dor lombar crônica por meio da radiofrequência \\ pulsada e a aplicação de esteroides na forma epidural. \\ Estudo comparativo
}

Treatment of chronic low back pain by means of pulsed radiofrequency and application of steroids in epidural form. Comparative study

\author{
Matín Tejeda-Barreras ${ }^{1}$ \\ Carlos Rodriguez-Celaya ${ }^{2}$ \\ Manuel A Santillana-Macedo ${ }^{3}$
}

\section{RESUMEN}

Objetivos: valorar el resultado clínico de mejoría de dolor y de escala funcional lumbar de radiofrecuencia pulsada, en comparación con aplicación de esteroides epidurales, en el tratamiento de pacientes con dolor lumbar crónico. Tipo de investigación: un ensayo clínico ciego y simple. Métodos: el estudio se realizó dese mayo de 2008 a julio de 2009. Fueron 40 pacientes, de los medios institucional y privado, de la ciudad de Hermosillo, Sonora, en el México, con dolor lumbar crónico. Se aplicó el cuestionario de Roland-Morris y la escala visual análoga de dolor, previa a tratamiento, y posterior al mismo. No se registraron pérdidas de pacientes en el seguimiento. Se analizaron los resultados de las diferentes variables (edad, sexo, ocupación, evolución, trabajo de carga, incapacidad, hallazgos de imagenología), estableciendo

\section{RESUMO}

Objetivos: avaliar o resultado clínico de melhoria da dor e da escala funcional lombar de radiofrequência pulsada, comparada com a aplicação de esteroides epidurais, no tratamento de pacientes com dor lombar crônico. Tipo de investigação: um ensaio clínico cego e simples. Métodos: o estudo foi realizado desde maio de 2008 a julho de 2009. Foram utilizados 40 pacientes, dos meios institucional e privado, da cidade de Hermosillo, Sonora, no México, com dor lombar crônico. Foi aplicado o questionário de Roland-Morris e a escala visual análoga de dor, com tratamento prévio e posterior a este. Não foram registradas perdas de pacientes no seguimento. Analisaram-se os resultados de diferentes variáveis (idade, sexo, ocupação, evolução, trabalho de carga, incapacidade, achados de imagenologia), estabelecendo

\section{ABSTRACT}

Objectives: to evaluate the clinical outcome of improving pain and functional scale lumbar pulsed radiofrequency, compared to the use of epidural steroids in the treatment of patients with chronic low back pain. Type of research: a randomized single and blind. Methods: the study was conducted from May, 2008 to July, 2009. There were 40 patients, through institutional and private means, in the city of Hermosillo, Sonora, Mexico, with chronic low back pain. The survey was conducted by Roland-Morris and visual analog scale of pain, with previous treatment, and subsequent to it. There were no losses of patients in the follow-up. We analyzed the results of different variables (age, sex, occupation, development, work load, disability, findings on imaging), and compared with the $\chi^{2}$ test. Results: they were

\footnotetext{
Trabajo realizado en el Hospital General de Zona \#2 Instituto Mexicano del Seguro Social - IMSS - Hermosillo, Sonora, México.

'Médico Especialista en Cirugía de Columna Vertebral en el Hospital General de Zona \#2, Instituto Mexicano del Seguro Social - IMSS - Hermosillo, Sonora, México.

${ }^{2}$ Médico Anestesiólogo Especialista en Clínica del Dolor del Instituto Mexicano del Seguro Social - IMSS - Hermosillo, Sonora, México.

${ }^{3}$ Médico Familiar con Maestría en Ciencias de la Salud Dolor del Instituto Mexicano del Seguro Social - IMSS - Hermosillo, Sonora, México.

Correspondencia: Martín Tejeda Barreras - Reforma n. 273 - Sur esquina Paseo San Miguel - Planta Baja 83280 - Hermosillo, Sonora -

E-mail:martintejedab@yahoo.com
} 
una comparación con la prueba de $\chi^{2}$. Resultados: fueron muy similares en ambos grupos de tratamiento $\left(\chi^{2}=2.8283, \mathrm{p}=0.093\right)$, con dos casos complicados por punción en el grupo de esteroides epidurales, sin casos complicados por el procedimiento de radiofrecuencia pulsada. Los valores de $\chi^{2}$ no muestran diferencias estadísticamente significativas, con valores de $\mathrm{p}$ de 0.09 a 0.9. Conclusión: la aplicación de radiofrecuencia pulsada es tan eficaz como la aplicación de esteroides epidurales para el manejo del dolor lumbar crónico.

\section{DESCRIPTORES: Dolor de} la región lumbar/terapia; Ondas radioeléctricas/uso terapéutico; Esteroides/uso terapéutico

uma comparação com a teste do $\chi^{2}$. Resultados: foram muito similares em ambos os grupos de tratamento $\left(\chi^{2}=2,8283, \mathrm{p}=0,093\right)$, com dois casos complicados por punção no grupo de esteroides epidurais, sem casos complicados pelo procedimento de radiofrequência pulsada. Os valores do $\chi^{2}$ não mostraram diferenças estatisticamente significativas com valores de $\mathrm{p}$ de 0,09 a 0,9. Conclusão: a aplicação da radiofrequência pulsada é tão eficaz quanto a aplicação de esteroides por via peridural no tratamento da dor lombar crônica.

DESCRITORES: Dor lombar/ terapia; Ondas de rádio/uso terapêutico; Esteróides/uso terapêutico very similar in both treatment groups $\left(\chi^{2}=2.8283, \quad p=0.093\right)$, with two cases complicated by puncture in the epidural steroid group, without cases complicated by use of pulsed radiofrequency. The values of $\chi^{2}$ showed no statistically significant differences, with p-values from 0.09 to 0.9. Conclusion: the application of pulsed radiofrequency is as effective as the application of epidural steroids for management of chronic low back pain.

KEYWORDS: Low back pain/ therapy; Radio waves/ therapeutic use; Steroids/ therapeutic use

\section{INTRODUCIÓN}

El dolor lumbar es una de las causas más frecuentes de consulta a nivel general y en la especialidad de Ortopedia y Traumatología, y la patología de gasto laboral más alto, y si bien la mayoría de los casos se resuelve al término de dos a tres semanas en promedio, su cronicidad origina grandes gastos y su tratamiento es difícil. Su manejo consiste en tratamientos de analgésicos y antiinflamatorios, así como sesiones de Medicina Física, donde se incluyen sesiones de ultrasonido, aplicación de calor, estimulación eléctrica y diversos esquemas de ejercicios.

En la actualidad, las inyecciones de esteroides epidurales han ganado popularidad en el tratamiento de pacientes con discopatías lumbares y radiculopatías, como origen de dolor lumbar crónico. Diversos estudios han documentado la reacción inflamatoria posterior a la lesión de disco lumbar $^{1}$, de manera que la aplicación de los esteroides epidurales parecería ser una elección lógica de tratamiento para este tipo de inflamación, la causa de su efecto sobre la estabilización de la membrana y en las fibras $\mathrm{C}$ antinociceptivas y su interferencia con los neuropéptidos y su actividad mediadora inflamatoria ${ }^{2,3}$.

Aunque las inyecciones de esteroides epidurales se han usado durante mucho tiempo en el tratamiento del dolor lumbar y los síntomas radiculares atribuidos a discopatía lumbar, continúa la controversia acerca de su eficacia, con literatura cargada de resultados contrastantes. Se han realizado estudios de revisión de ensayos clínicos aleatorios (15 ensayos), valorando el uso de las inyecciones epidurales en pacientes con dolor lumbar y ciatalgia, notando que cerca de la mitad de los estudios reportaban resultados favorables, mientras que la otra mitad reportaba resultados negativos. Los autores concluyeron que la eficacia de estas inyecciones aún queda por establecerse, y que si lo había, los beneficios eran de corta duración ${ }^{4,5}$.

En cuanto a la radiofrecuencia, es un procedimiento en donde un electrodo es guiado hacia las facetas articulares, principalmente. El electrodo es valorado radiográficamente para asegurar que la orientación sea paralela al curso estimado anatómico de lo nervio de la rama medial.

Posteriormente, el electrodo es calentado a 42 grados centígrados, y si la articulación es el origen del dolor, la termocoagulación eliminaría el dolor.

Dreyfuss et al. realizaron este procedimiento en lesiones de facetas en un grupo de pacientes. El $60 \%$ de sus pacientes reportaron por lo menos un $90 \%$ de reducción de dolor, y el $87 \%$ reportaron al menos un $60 \%$ de mejoría de dolor a un seguimiento de 12 meses $^{6}$.

En la actualidad, los resultados con ambas técnicas son controversiales, por lo que es necesario realizar estudios de ensayo clínico aleatorio para aportar más información de la mejoría que puedan lograr los pacientes, ya que la mayoría de los estudios reportados, hasta la fecha, es con base en serie de casos o estudios retrospectivos, con un número limitado de pacientes ${ }^{7,8}$.

Teniendo en cuenta lo anterior, se decidió realizar un estudio clínico aleatorio, ciego y simple, comparando las dos formas de tratamiento, con un grupo de pacientes sometidos a aplicación de radiofrecuencia pulsada y otro grupo de pacientes a quienes se aplicaron esteroides en forma epidural. 


\section{MÉTODOS}

\section{Tipo de investigación}

Se trata de una investigación de tipo ensayo clínico controlado, ciego y simple. Es un estudio de mínimo riesgo, comparativo de dos métodos de tratamiento, estableciendo la comparación con la prueba estadística de $\chi^{2}$.

\section{Población de estudio}

La población consistió en 40 pacientes adultos, sin distinción de género, de la consulta externa, del Servicio de Ortopedia y Traumatología del Hospital General de Zona \#2 del Instituto Mexicano del Seguro Social, así como pacientes de medio privado, del hospital CIMA, en la ciudad de Hermosillo, Sonora, en México, desde mayo de 2008 hasta julio de 2009, con patología de dolor lumbar de más de tres meses de evolución, y hasta dos años, valorada en escala visual análoga de dolor, con limitación de actividades de la vida diaria y limitación laboral, determinados en la escala funcional lumbar de Roland Morris. Los pacientes tenían tratamiento oral o parenteral previo de analgésicos/antiinflamatorios, de tipo no esteroideo, por un tiempo mínimo de tres meses, y tratamiento de Medicina Física y Rehabilitación, consistente en aplicación de ultrasonido, electroestimulación, calor local superficial y profundo, en sesiones de por lo menos una hora diaria durante un tiempo mínimo de tres semanas, sin mejoría a estos tratamientos, con origen de dolor identificado por cuadro clínico e imagenología (rayos X simples de columna lumbosacra, tomografía axial computarizada, o resonancia magnética nuclear), en facetas, discos, o forámenes intervertebrales de la columna lumbar.

\section{Criterios de selección}

\section{Criterios de inclusión}

- Pacientes adultos, sin distinción de género, con dolor de origen lumbar de un mínimo de tres meses hasta dos años de evolución;

- Tratamiento previo por un tiempo mínimo de tres meses con medicamentos tipo analgésicos y antiinflamatorios no esteroideos, y Medicina Física, sin mejoría;

- Valoración de dolor con escala visual análoga;

- Valoración de escala funcional de Roland-Morris para columna lumbar.

Criterios de exclusión

- Expediente incompleto;

- Patología agregada de neuropatía de origen metabólico;

- Ganancia secundaria económica (pacientes con cuadros repetitivos con incapacidad prolongada);

- Discordancia de cuadro clínico e imagenología;

- Cirugía previa en columna lumbar.

Criterios de eliminación

- Pérdida de expediente clínico;

- Tratamientos agregados a la radiofrecuencia;

- Realización de tratamiento quirúrgico.

\section{Análisis estadístico}

La captura de los datos se realizó en hojas de Excel de programa Windows XP, versión 2007, en computadora laptop marca Dell del año 2007, determinando los datos estadísticos básicos descriptivos de promedios, valores mínimos y máximos de las variables, y el procesamiento de datos se llevo a cabo en el programa estadístico STATA, versión 9, realizando el análisis estadístico en computadora de marca Apple Macintosh del año 2007, con procesador Safari X, y posteriormente convertidos al programa Windows. Los resultados de las frecuencias y los promedios fueron comparados con una prueba de $\chi^{2}$.

\section{RESULTADOS}

En el Grupo de Radiofrecuencia Pulsada, fueron 8 hombres y 12 mujeres, con un promedio de edad de 50 años (desde 38 a 71 años), nueve de estos pacientes no laboran, tres son empleados, un trabajador de campo, y cuatro profesionistas, y tres más fueron trabajadores no calificados. Cuatro de estas personas realizaban labores de carga. Ocho de estas personas se encontraban incapacitados (desde 3 hasta 11 meses de incapacidad). Se detectaron alteraciones de depresión en 3 de estos 20 pacientes. El mecanismo de lesión fue espontáneo en 12 de ellos, 4 por esfuerzo de carga, y otros 4 por caída. El tiempo de evolución de su dolor varió de 4 hasta 18 meses. En las radiografías simples, en este grupo de estudio, se encontraron cambios degenerativos en 15 de ellos, en otros 3 se notó disminución de altura, y en 2 se encontraron radiografías sin datos patológicos.

La tomografía axial computarizada se realizó en 18 de estos pacientes, predominando las artrosis de facetas en L4L5-S1 en nueve casos, en otros cinco se encontraron alteraciones en los discos, con presencia de gas en los mismos, y en tres casos se encontró estenosis de menos de $30 \%$ del conducto vertebral, y hubo un caso con lesiones mixtas, con degeneración discal y artrosis facetaría de L4/L5. Se realizaron cuatro estudios de resonancia magnética, los cuales fueron complementos o sustituciones del estudio de la tomografía, encontrando presencia de degeneración discal con disco negro en tres casos y un caso de artrosis facetaría. Todos los pacientes recibieron tratamiento previo de diferentes tipos de AINES, y 16 de ellos recibieron además complemento de Medicina Física de dos hasta seis semanas.

En el grupo de aplicación de esteroides epidurales, fueron 13 mujeres y 7 hombres, con un promedio de edad de 43.1 años (desde 21 hasta 68 años de edad). En cuanto a su ocupación, en este grupo de pacientes, seis no laboran, cinco son empleados, dos fueron profesionistas, dos trabajadores de campo, y cinco trabajadores no calificados; entre los pacientes que laboran, cinco realizan labores de carga. También entre las personas que laboran, nueve se encontraban incapacitadas (desde 4 hasta 12 meses). En lo referente a alteraciones de depresión, se encontraron en 2 de los 20 pacientes. El mecanismo de lesión fue espontáneo en diez casos, en cinco fue por esfuerzo de carga, y cinco más por caída. El tiempo de evolución de dolor varió 
de 3 hasta 12 meses. Las radiografías simples en cinco casos no mostraron alteraciones, en site se encontró disminución de la altura intersomática, y en ocho se encontraron cambios degenerativos. La tomografía axial computarizada se realizó en 17 casos, encontrando artrosis facetaría en seis casos, siete con proceso de degeneración discal, y en dos más con estenosis de conducto neurológico de menos de un $30 \%$ del diámetro del conducto, y dos casos de protrusión discal de menos de un $20 \%$ del diámetro del conducto. Se realizó estudio de resonancia magnética de la columna lumbosacra en siete casos como complemento o sustitución de la tomografía axial, encontrando dos casos de protrusión discal, dos casos con hallazgos de disco negro, dos con estenosis de menos de un $20 \%$ del conducto neurológico, y artrosis facetaría en uno de los casos. Los resultados se muestran en la Tabla 1.

\section{TABLA 1 - Resumen de los resultados (Excel)}

\begin{tabular}{|c|c|c|}
\hline Variable & Radiofrecuencia & $\begin{array}{l}\text { Esteroides } \\
\text { epidurales }\end{array}$ \\
\hline \multicolumn{3}{|l|}{ Género } \\
\hline Masculino & 8 & 7 \\
\hline Femenino & 12 & 13 \\
\hline \multicolumn{3}{|l|}{ Edad } \\
\hline Mínima & 38 & 38 \\
\hline Máxima & 71 & 71 \\
\hline Promedio & 50 & 50 \\
\hline \multicolumn{3}{|l|}{ Trabajadores } \\
\hline Sí & 11 & 14 \\
\hline No & 9 & 6 \\
\hline \multicolumn{3}{|l|}{ Incapacitados } \\
\hline Promedio & 8 & 9 \\
\hline Variación & 3 a 11 meses & 4 a 14 meses \\
\hline \multicolumn{3}{|l|}{ Mecanismo lesión } \\
\hline Carga & 4 & 5 \\
\hline Caída & 4 & 5 \\
\hline Espontáneo & 12 & 10 \\
\hline Tiempo de evolución & 4 a 18 meses & 3 a 12 meses \\
\hline \multicolumn{3}{|l|}{ Rayos X } \\
\hline Degenerativos & 15 & 8 \\
\hline Disminución de altura & 3 & 7 \\
\hline Sin lesión ósea & 2 & 5 \\
\hline \multicolumn{3}{|l|}{$\begin{array}{l}\text { Tomografía axial } \\
\text { computadorizada }\end{array}$} \\
\hline Artrosis facetas & 9 & 6 \\
\hline Discopatía & 5 & 7 \\
\hline Estenosis & 3 & 2 \\
\hline Otras & 1 & 2 \\
\hline \multicolumn{3}{|l|}{$\begin{array}{l}\text { Resonancia magnética } \\
\text { simple }\end{array}$} \\
\hline Artrosis facetas & 1 & 1 \\
\hline Discopatía & 3 & 3 \\
\hline Estenosis & 0 & 0 \\
\hline Protrusión & 0 & 0 \\
\hline
\end{tabular}

En este grupo de pacientes, todos recibieron tratamiento con diferentes tipos de medicamentos analgésicos/antiinflamatorios, y además complemento con tratamiento de Medicina Física en 19 de los 20 casos, en un promedio de tres semanas (variando de dos hasta seis semanas).

Se revisaron los resultados y se compararon los dos grupos, utilizando prueba estadística de $\chi^{2}$, encontrando al comparar los resultados de mejoría de dolor y de escala funcional, una $\chi^{2}$ de $2.8283(\mathrm{p}=0.093)$, es decir, sin diferencia estadísticamente significativa. Al comparar los resultados por sexo, se encontró una $\chi^{2}$ de $0.6734(\mathrm{p}=0.412)$; al comparar con el tipo de trabajo, se encontró un valor de $\chi^{2}$ de $1.350(\mathrm{p}=0.851)$; al comparar los dos grupos con el hecho de realizar labores de carga, se encontró una $\chi^{2}$ de 0.1347 ( $\mathrm{p}=0.714)$; cuando se realizó la comparación con las personas que estaban incapacitadas, se encontró un valor de $\chi^{2}$ de 0.7533 ( $\left.\mathrm{p}=0.385\right)$; al comparar los mismos parámetros de dolor y escala funcional con el mecanismo de lesión, la $\chi^{2}$ fue de $0.0041(\mathrm{p}=0.998)$; al comparar con los hallazgos de rayos $\mathrm{X}$, los resultados de dolor y escala funcional, se encontró un valor de $\chi^{2}$ de $3.4160(\mathrm{p}=0.181)$, y al comparar los resultados de ambos los grupos en lo referente a lo hallazgos de tomografía axial computarizada se encontró una $\chi^{2}$ de $5.0128(\mathrm{p}=0.082)$, y en lo referente a la resonancia magnética, la $\chi^{2}$ fue de $1.7143(\mathrm{p}=0.424)$. Finalmente, se compararon los resultados con las personas que presentaban cuadro de depresión, encontrando una $\chi^{2}$ de $0.0577(\mathrm{p}=0.810)$. Los resultados de la $\chi^{2}$ en todas las variables comparadas no muestran diferencias estadísticamente significativas, con valores de de 0.09 a 0.9 .

En el grupo de aplicación de esteroides epidurales, se reportaron, en dos casos, persistencia de dolor en el sitio de aplicación del esteroide por cuatro a seis semanas, siendo uno de los casos una punción accidental de saco dural, tratado con parche hemático, y resuelta la complicación a las seis semanas. Se encontraron, además, persistencia de dolor en las mismas condiciones previas al tratamiento en tres casos, siendo pensionados finalmente en sus trabajos, mientras que en el grupo de radiofrecuencia pulsada, en dos casos se mantuvo el dolor en las mismas condiciones previas al tratamiento. Finalmente, fueron pensionados.

En ambos los grupos, los pacientes en los cuales se detectaron alteraciones psicológicas de tipo depresivas en el seguimiento fueron enviados a continuar tratamiento en Psiquiatría.

De acuerdo a los resultados anteriormente expuestos, se aprecia que ambos métodos de tratamiento eran igualmente eficaces para el tratamiento del dolor lumbar crónico, sin poder establecer una superioridad en cuanto a la eficiencia de uno sobre el otro (Figuras 1 y 2). Se compararon además, con el mismo método, los diferentes grupos de edad, sexo, las ocupaciones, el realizar labores de carga, el contar con incapacidad, el tiempo de evolución, los hallazgos de imagenología, y la presencia de cuadro depresivo, donde en todos estos rubros se aprecian los mismos resultados. 


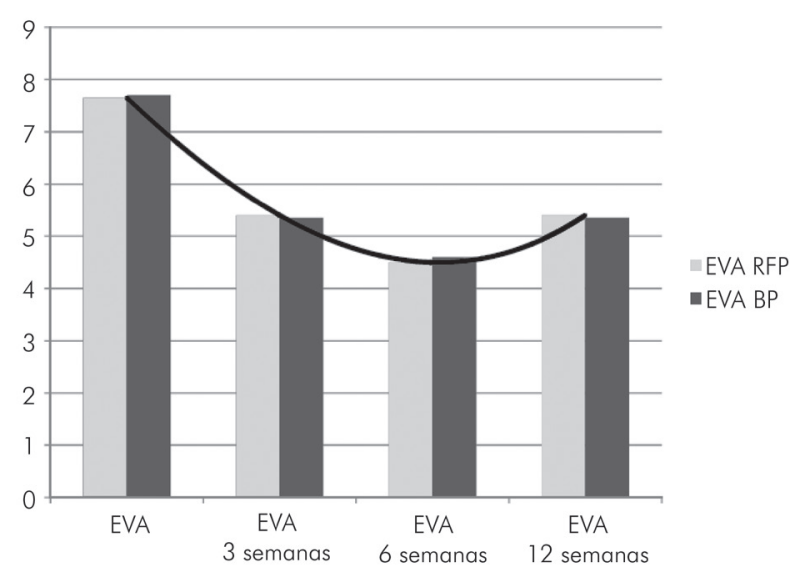

Figura 1. Comparativa de escala visual de dolor en ambos grupos.

EVA caso - dolor en grupo de aplicación radiofrecuencia pulsada; EVA control - dolor en grupo de aplicación esteroides epidurales.

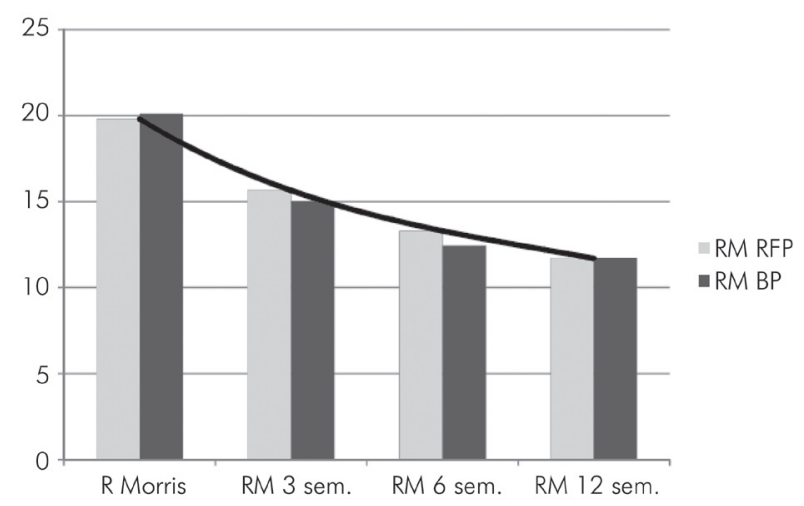

Figura 2. Comparativa de escala visual de dolor en ambos grupos.

EVA caso - dolor en el grupo de aplicación de radiofrecuencia pulsada; EVA control - dolor en el grupo de aplicación de esteroides epidurales.

\section{DISCUSIÓN}

En los últimos 20 años, el dolor lumbar se ha convertido en una de las entidades clínicas de más difícil tratamiento, principalmente porque los mecanismos de producción de dolor son mal comprendidos, tenemos poco conocimiento de ellos, y la correlación clínica con estudios complementarios es difícil de se establecer.

En el presente estudio, se cumplió el objetivo de comparar los dos métodos de tratamiento, ya que encontramos una efectividad similar de mejoría de dolor y de cambio favorable en la escala funcional de columna lumbar, tanto con el tratamiento en la radiofrecuencia pulsada como en la aplicación de esteroides epidurales. Los resultados pueden considerarse consistentes con la metodología utilizada, sin encontrar controversias ni complicaciones desde el punto de vista bioético. En los estudios realizados previamente, acerca de la aplicación de esteroides epidurales, los resultados son discordantes, reportando en los casos favorables una mejoría del 40 a $60 \%$ y siendo en corto plazo el resultado ${ }^{5,9}$. Se ha reportado, además, diversas técnicas para aplicación de esteroides epidurales, lo cual también influye en los resultados 9 El presente estudio da una mejoría de dolor de más del 60\% en 12 de los casos, en los cuales se aplicaron esteroides epidurales, lo que es concordante con los estudios publicados previamente. También se refieren complicaciones potenciales con la aplicación de esteroides epidurales, desde desgarros de saco dural (ocurrieron dos punciones accidentales en el presente estudio), hematoma intradural, hasta infecciones del conducto neurológico, los cuales afortunadamente no se presentaron. Estas complicaciones, aunque su frecuencia es baja (menos del 5\% en series largas) ${ }^{3}$, constituyen un riesgo con la aplicación de esteroides epidurale.

En lo referente a la aplicación de radiofrecuencia pulsada, se habían publicado series cortas desde su inicio, con 20 a 25 pacientes ${ }^{10}, \mathrm{y}$ con el tiempo se han ido presentando series con un mayor número de casos, aunque la mayoría son estudios retrospectivos, o series de casos, en un número de 12 artículos en los últimos diez años, con resultados clínicos de mejoría en promedio siendo esta mejoría del 70 a $80 \%^{6-8}$. En el presente estudio, la mejoría con la aplicación de radiofrecuencia pulsada fue de bajar de una escala de 7.5 a 4.5 en promedio, lo que significa una disminución del $60 \%$, por debajo de lo reportado en la literatura. Debemos de mencionar que la radiofrecuencia pulsada, hasta la fecha, es un tratamiento que si bien se establece de mínima invasividad - al igual que la aplicación de esteroides -, no presenta efectos colaterales reportados como en el caso de los esteroides aplicados epiduralmente (desgarros de saco dural, punción de raíces nerviosas, sangrado, extravasación del medicamento). Estos en manos expertas representan un mínimo porcentaje, no deja de existir, lo cual convierte al procedimiento de aplicación de radiofrecuencia pulsada en un método de tratamiento seguro y con una eficacia comparable a la de esteroides epidurales.

Este estudio es de utilidad, debido a que relaciona un método diferente al establecido, comparando en forma aleatoria los resultados obtenidos, encontrando mejoría de dolor y de escala funcional, en forma similar al Grupo Control. Se compararon, además, las posibles causas de los malos resultados en ambos grupos, como fueron la incapacidad prolongada y en aquellos pacientes en quienes se detectó alteraciones emocionales de tipo depresión, encontrando igual proporción en ambos grupos, lo cual es concordante en estudios similares de tratamiento de dolor lumbar crónico, tanto de métodos quirúrgicos, como en tratamientos no quirúrgicos.

\section{CONCLUSIONES}

En este estudio no hubo diferencias estadísticamente significativas en cuanto al resultado de mejoría de dolor medido en la escala visual análoga y en el cuestionario funcional de Roland-Morris, entre el tratamiento de radiofrecuencia pulsada y la aplicación de esteroides epidurales. 
La aplicación de radiofrecuencia pulsada es tan eficaz como la aplicación de esteroides epidurales para el manejo del dolor lumbar crónico.

En términos de seguridad, la radiofrecuencia pulsada demuestra una menor tasa de complicaciones en relación con la aplicación de esteroides epidurales.

El período de tiempo en que se efectuó el estudio es de corto plazo, por lo que no es posible extrapolar los resultados a largo plazo.
Existen factores complejos, cuya medición es difícil, en la generación del dolor crónico, como la ganancia secundaria de tipo económica, o los factores psicológicos, cuya determinación no ha sido posible establecer como primarios o secundarios a la presencia de dolor crónico.

La muestra de este estudio es relativamente pequeña, por lo que se debe continuar realizando estudios, buscando alternativas de tratamiento para tratar el dolor lumbar crónico.

\section{REFERENCIAS}

1. Takahashi H, Suguro T, Okazima Y, Motegi M, Okada Y, Kakiuchi $\mathrm{T}$. Inflammatory cytokines in the herniated disc of the lumbar spine. Spine (Phila Pa 1976). 1996;21(2):218-24.

2. Johansson A, Hao J, Sjölund B. Local corticosteroid application blocks transmission in normal nociceptive C-fibres. Acta Anaesthesiol Scand. 1990;34(5):335-8.

3. Brodke, Darrel MD, Ritter, Stephen MD. Non operative management of low back pain. J Bone Joint Surg A. 2004;86(8):1810-18.

4. Koes BW, Scholten RJ, Mens JM, Bouter LM. Epidural steroid injections for low back pain and sciatica. Pain . 1995;63(3)279-88.
5. Weinstein SM, Herring SA; NASS. Lumbar epidural steroid injections. Spine J. 2003;3(3 Suppl):37-44S.

6. Dreyfuss P, Halbrook B, Pauza K, Joshi A, McLarty J, Bogduk N. Efficacy and validity of radiofrequency neurotomy for chronic lumbar zygapophysial joint pain. Spine. 2000;25(10):1270-7.

7. Racz GB, Ruiz-Lopez R. Radiofrequency procedures. Pain Pract. 2006;6(1):46-50.

8. Lindner R, Sluijter ME, Schleinzer W. Pulsed radiofrequency treatment of the lumbar medial branch for facet pain: a retrospective analysis. Pain Med. 2006;7(5):435-9.
9. Snarr J. Risk, benefits and complications of epidural steroid injections: a case report. AANA J. 2007;75(3):183-8.

10. Sluijter ME, Metha M. 1981. Treatment of back and neck pain by percutaneous thermal lesions in persistent pain. London: Academic Press; 1981. p. 141-79.

\section{Correspondencia}

Martín Tejeda Barreras Reforma n. 273 - Sur esquina Paseo San Miguel - Planta Baja 83280 Hermosillo, Sonora

E-mail:martintejedab@yahoo.com 